\title{
Evaluation de la formation postgraduée
}

\author{
Bernadette Sütterlina, Lea Christina Burgermeister ${ }^{b}$, Michael Siegrist ${ }^{c}$, Werner Bauer ${ }^{d}$ \\ ${ }^{a}$ Dr sc., Senior Researcher; ${ }^{b}$ Lic. phil., collaboratrice scientifique; ${ }^{c}$ Prof. Dr phil., docent Consumer Behavior, ETH Zurich; ${ }^{d}$ Dr med., président de I'ISFM
}

Sur mandat de la Confédération, l'Institut suisse pour la formation médicale postgraduée et continue (ISFM) décerne les titres de spécialiste et se charge d'organiser la formation postgraduée avec les sociétés de discipline médicale. Même si la formation postgraduée jouit d'une excellente réputation, sa qualité doit être continuellement évaluée. Ce contrôle de qualité est mené dans le cadre de l'évaluation du concept de formation de chaque établissement de formation postgraduée, puis de visites sur place et de l'enquête annuelle auprès des médecins en formation postgraduée. En 2014, c'était la $18^{\mathrm{e}}$ édition de cette enquête menée sur mandat de l'ISFM.

En novembre 2014, le responsable de chaque établissement de formation postgraduée reconnu a reçu un rapport comportant les retours détaillés sur les résultats relatifs à son établissement de formation. Ce rapport indique également à titre de comparaison la moyenne des évaluations de tous les établissements de formation postgraduée de la spécialisation correspondante (pour les principales disciplines, le calcul de la valeur comparative est différencié selon la taille de l'établissement de formation). Un récapitulatif de l'ensemble des résultats (graphique en toile d'araignée) des différents établissements de formation postgraduée est publié sur le site Internet de l'ISFM.

L'enquête annuelle a pour objectif de contribuer à la garantie de la qualité de la formation postgraduée. Huit dimensions d'évaluation sont recensées: appréciation générale, transmission de compétences professionnelles, culture d'apprentissage, culture d'encadrement, culture de l'erreur, culture de la prise de décision, culture d'entreprise et evidence based medicine. Hormis cette partie générale qui ne varie guère d'une année sur l'autre, le questionnaire comprend chaque année une partie variable. Les médecins y sont interrogés sur des sujets d'actualité liés à leur formation postgraduée. En 2014, cette partie comportait des questions sur l'impact de l'introduction à l'échelle nationale du système SwissDRG ainsi que sur les déterminants du choix du titre de spécialiste. Le système DRG avait déjà fait l'objet de l'enquête 2011 avant son intro- duction. La reprise de ce thème dans l'enquête 2014 a donc permis une comparaison directe entre les effets escomptés avant l'introduction du système DRG et les changements perçus après son introduction.

Pour préparer cette enquête, un recensement statistique des postes de formation postgraduée est effectué chaque année au printemps auprès des responsables des établissements de formation postgraduée afin d'obtenir des informations sur les médecins en formation qu'ils emploient. Les responsables sont également interrogés sur des thèmes d'actualité dans le cadre de ce recensement. A l'instar de l'enquête menée auprès des médecins en formation postgraduée, le questionnaire destiné aux responsables des établissements de formation postgraduée comportait également en 2014 des questions sur l'impact de l'introduction à l'échelle nationale du système SwissDRG. Ces questions avaient déjà été posées aux responsables des établissements en 2011. Le présent article donne un aperçu des principaux résultats de l'enquête 2014; des chiffres comparatifs issus de l'enquête de 2011 seront également présentés dans la mesure du possible.

\section{Méthodologie}

\section{Participants et réalisation}

1275 établissements de formation postgraduée ont participé à l'enquête 2014 et un total de 10328 questionnaires ont été envoyés à l'attention des médecins en formation postgraduée. Les responsables des établissements de formation postgraduée étaient en effet chargés de les distribuer aux médecins en formation qu'ils avaient déclarés. Les questionnaires remplis ont été retournés, dans une enveloppe retour personnelle, directement et de façon anonyme à la chaire Consumer Behavior de l'ETH Zurich chargée de la réalisation de cette enquête annuelle. Le recensement des données s'est étalé sur une période de trois mois environ (de début juillet à mi-octobre 2014). Le taux de réponse a atteint $66 \%(\mathrm{~N}=6831)$. Cette valeur est comparable au taux de réponse des sondages précédents [1]. 
Dans le cadre du recensement statistique des postes de formation postgraduée effectué au printemps, les responsables des établissements ont reçu un lien vers un bref questionnaire en ligne. 1370 responsables y ont répondu (94\%). Pour l'analyse des résultats présentés ci-après, seules les réponses des responsables d'établissements ayant indiqué qu'ils utilisaient le système SwissDRG ont été prises en compte $(\mathrm{N}=1008)$.

\section{Questionnaire}

Le questionnaire destiné aux médecins en formation postgraduée était disponible en trois langues. Les questions portant sur les dimensions d'évaluation, appréciation générale, culture d'apprentissage, culture d'encadrement, culture de l'erreur, culture de la prise de décision, culture d'entreprise et evidence based medicine ont été formulées sous forme de déclarations. Les participants ont été priés d'indiquer dans quelle mesure une déclaration était applicable à leur cas. Dans la partie consacrée aux compétences professionnelles, les compétences concrètes ont été énumérées, et les participants devaient évaluer dans quelle mesure l'établissement de formation postgraduée contribuait à leur développement.

La partie variable comportait un module de questions sur l'introduction à l'échelle nationale du nouveau financement hospitalier/DRG et un autre module sur le choix de la spécialisation médicale. Le module DRG a permis de recenser l'opinion des médecins en formation postgraduée sur l'impact du système DRG sur leur travail. On leur a également demandé dans quelle mesure le système DRG tenait compte, à leurs yeux, des différents groupes de patients. Ces questions ont été formulées sous forme de déclarations, et les participants ont été priés d'indiquer leur approbation. Certaines questions sur le nouveau financement hospitalier/DRG avaient déjà été posées avant l'introduction à l'échelle nationale dans l'enquête 2011. Cela a donc per-



Figure 1: Valeurs moyennes (y compris intervalle de confiance de 95\%) des huit dimensions d'évaluation (plus la valeur est élevée, plus l'évaluation est positive). Seules les réponses des médecins en formation postgraduée exerçant depuis au moins 2 mois dans l'établissement ont été prises en compte. mis de comparer les réponses faites par les médecins en formation avant et après l'introduction du système DRG. A l'instar de l'enquête menée auprès des médecins en formation postgraduée, les responsables des établissements de formation postgraduée ont également été interrogés sur l'impact de l'introduction du système DRG dans le cadre des recensements statistiques en 2011 et 2014.

Le module sur le choix de la spécialisation médicale comportait d'une part des questions sur les raisons du choix. Dix aspects différents ont été énumérés; les médecins en formation postgraduée devaient évaluer dans quelle mesure chacun de ces éléments influençait ou avait influencé leur choix. La seconde partie de ce module portait sur le moment du choix. Dans une première question, les participants ont été priés d'indiquer à quel moment ils avaient pris leur décision et dans une seconde, s'ils avaient changé de voie en cours de formation.

\section{Résultats}

\section{Evaluation générale des établissements de formation postgraduée}

Dans l'ensemble, les médecins en formation jugent positive la situation dans les établissements de formation postgraduée. Les établissements de formation postgraduée qui présentent un faible nombre de médecins en formation s'en tirent mieux comme toujours [1]. La figure 1 représente les huit différentes dimensions d'évaluation. La culture de l'erreur, la culture de la prise de décision et la culture d'entreprise obtiennent les meilleurs résultats, tandis que l'evidence based medicine est un peu en retrait.

\section{Nouveau financement hospitalier/DRG}

\section{Réponses transmises par les responsables des établissements de formation postgraduée} Impact sur la formation postgraduée: En 2011, 51\% des responsables interrogés considéraient que l'introduction du système DRG menaçait des places de formation postgraduée dans leur établissement. Trois ans plus tard, seulement $4 \%$ d'entre eux indiquaient que l'introduction du système DRG avait effectivement réduit le nombre de places de formation postgraduée. Avant l'introduction, 29\% des responsables interrogés ont indiqué qu'ils envisageaient de remplacer les médecins en formation postgraduée par des médecins hospitaliers en perspective de l'introduction du système DRG tandis que seulement 3\% d'entre eux indiquent en 2014 avoir effectivement pris une telle mesure. Toutefois, 




Figure 2: Conviction que le système DRG ne tient pas compte, du point de vue médical, de certains groupes de patients avant et après I'introduction du système DRG, selon les responsables des établissements de formation postgraduée (2011: $N=1070 ; 2014: N=1008$ ) et selon les médecins en formation postgraduée (2011: $N=6281 ; 2014: \mathrm{N}=6831$ ).

19\% d'entre eux indiquent encore en 2014 qu'ils envisagent de remplacer des médecins en formation postgraduée (supplémentaires) par des médecins hospitaliers.

Impact sur les patients: L'impact du système DRG sur les patients fait presque l'unanimité chez les responsables interrogés. Avant l'introduction du système DRG, la majorité des responsables (83\%) considéraient que le système DRG ne tenait pas compte, du point de vue médical, de certains groupes de patients. Après l'introduction de SwissDRG, cette crainte semble malheureusement se confirmer puisque $78 \%$ des personnes interrogées partagent toujours cet avis en 2014. L'opinion n’a donc pratiquement pas évolué.

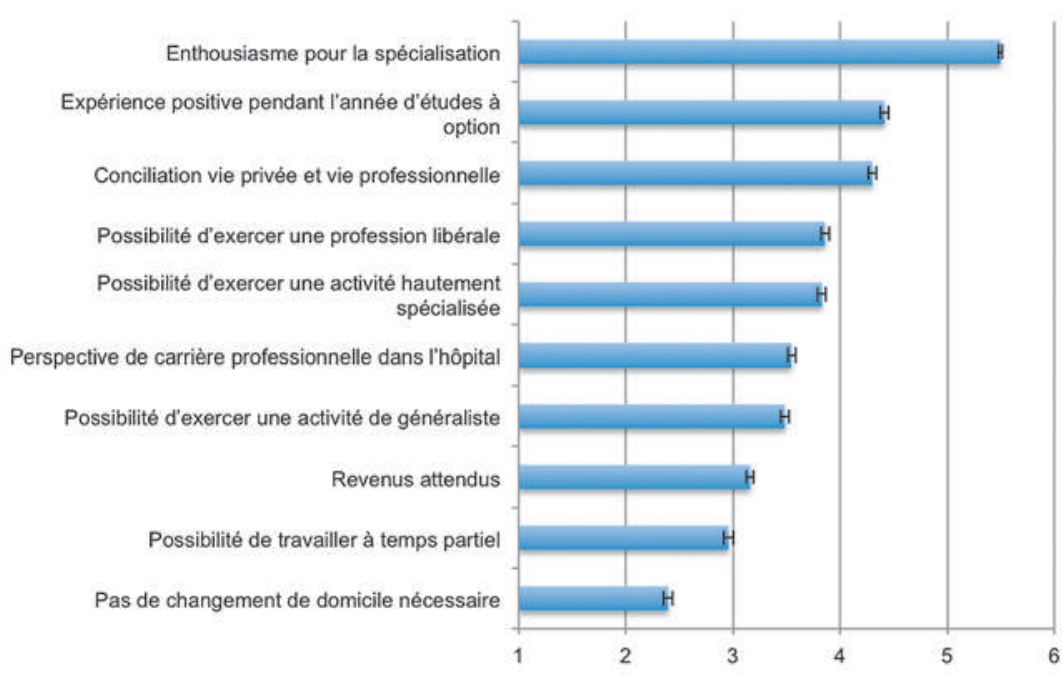

Figure 3: Facteurs déterminants du choix de la spécialisation ( $1=$ absolument aucune influence, 6 = très forte influence), triés en fonction de leur incidence. Les valeurs moyennes et les intervalles de confiance de $95 \%$ correspondants sont représentés.

\section{Réponses transmises par les médecins en} formation postgraduée

Impact sur la formation postgraduée: L'impact de l'introduction à l'échelle nationale du nouveau financement hospitalier/DRG sur la situation de la formation postgraduée est jugé négatif, mais moins négatif que ce que l'on craignait. Alors qu'en 2011, 71\% des médecins en formation postgraduée redoutaient que l'introduction du nouveau financement hospitalier/DRG ait un impact négatif sur leur travail, ils sont encore $54 \%$ de cet avis en 2014.

Impact sur les patients: Les médecins en formation postgraduée sont convaincus à $89 \%$ en 2011 et à $81 \%$ en 2014 que le système DRG ne tient pas compte, du point de vue médical, de certains groupes de patients - un avis plus largement répandu donc que chez les responsables d'établissements de formation postgraduée. Les résultats sont représentés en détail dans la figure 2.

\section{Choix de la spécialisation}

Motivations du choix de la spécialisation: L'enthousiasme pour une discipline médicale est de loin le principal aspect pris en compte dans le choix d'une spécialisation. Cependant, les expériences positives faites pendant l'année d'études à option ainsi que la conciliation de la vie privée et de la vie professionnelle jouent également un rôle important. En revanche, un éventuel changement de domicile, la possibilité de travailler à temps partiel et les revenus attendus revêtent une moindre importance. Les résultats concernant les facteurs déterminants du choix du titre de spécialiste sont représentés dans la figure 3 .

L'importance des différents aspects varie selon les spécialisations. Si l'on considère les sept disciplines les plus fréquentes, l'enthousiasme pour la spécialisation arrive en tête de classement dans toutes les sept disciplines. L'année d'études à option occupe, elle aussi, l'un des trois premiers rangs, sauf pour la médecine interne générale et la psychiatrie. Un changement de domicile et les revenus sont relayés aux trois derniers rangs dans toutes les spécialisations, sauf en chirurgie orthopédique, seule discipline pour laquelle la rémunération est un facteur déterminant de moyenne importance. L'importance de la conciliation de la vie privée et de la vie professionnelle varie fortement selon les différentes spécialisations. Elle joue en effet un rôle majeur dans le choix des spécialisations de médecine interne générale, d'anesthésiologie et de psychiatrie, mais est d'une pertinence moyenne dans le choix des autres disciplines. (Les résultats détaillés pour les sept spécialisations les plus fréquentes peuvent être consultés en ligne sous www.bullmed.ch $\rightarrow$ Numéro actuel $\rightarrow$ Tableau 1.) 




Figure 4: Moment du choix d'une spécialisation, selon les sept disciplines les plus fréquentes.
Correspondance: Institute for Environmental Decisions (IED) Consumer Behavior ETH Zurich CHN I 76.3 Universitätstrasse 22 CH-8092 Zurich
Moment du choix de la spécialisation. La moitié seulement des médecins en formation ont choisi leur spécialisation avant le début de la formation postgraduée. $28 \%$ d'entre eux ont déjà pris leur décision pendant ou après l'année d'études à option tandis que $23 \%$ ont déjà choisi leur spécialisation avant cette même année. Environ un tiers (36\%) des médecins ont opté pour une spécialisation pendant leur formation postgraduée et une très petite proportion (4\%) qu'après l'obtention de leur premier titre de spécialiste. Les autres participants à l'enquête n'avaient pas encore choisi de spécialisation ou n'ont fourni aucune information à ce sujet. Le moment de ce choix varie considérablement selon le titre de spécialiste visé (fig. 4). Pour la médecine interne générale, l'anesthésiologie et la psychiatrie, la majorité des futurs spécialistes n'ont pris leur décision qu'au cours de leur formation postgraduée. Il est frappant de constater qu'une grande partie des médecins visant le titre de spécialiste en pédiatrie avait déjà pris sa décision avant l'année d'études à option.

\section{Discussion}

L’enquête 2014 révèle que la grande majorité des médecins en formation postgraduée ayant participé au sondage sont globalement satisfaits de la formation postgraduée reçue et évaluent positivement leur établissement de formation. Pour les responsables des établissements de formation postgraduée, cette enquête est un instrument précieux pour établir un bilan périodique de la qualité du cursus proposé, telle qu'elle est perçue par les médecins en formation. Elle leur permet d'identifier d'éventuelles lacunes, de déceler à temps des développements problématiques et de prendre des mesures d'amélioration ciblées. Les rares cas qui présentent des résultats globaux clairement inférieurs à la moyenne sont signalés à la société de discipline médicale concernée qui sera chargée d'examiner la situation et, surtout, de prendre les mesures nécessaires en cas de récidive (par ex. visite sur place ou remise en cause de la reconnaissance).

Le choix précoce d'une spécialisation de la part des futurs spécialistes fait aujourd'hui l'objet d'un débat dans le but d'améliorer la planification et la gestion de l'offre dans les différentes catégories de spécialistes en vue de garantir les soins médicaux à l'avenir. L'évaluation des réponses concernant le moment du choix de la spécialisation montre que, dans certaines disciplines comme la médecine interne générale et la psychiatrie, cette décision est prise assez tardivement. On peut donc s'interroger si le fait d'obliger les médecins en formation postgraduée à choisir rapidement une spécialisation leur permettra d'opter véritablement pour la discipline répondant au mieux à leur intérêt et leurs aptitudes à long terme.

L'introduction du système DRG a suscité de nombreuses inquiétudes concernant son impact négatif possible sur l'exercice de l'activité médicale, la formation postgraduée et la prise en charge des patients. Comme le révèlent les résultats de l'enquête, l'introduction du système DRG n'a pas eu à ce jour des conséquences négatives aussi graves que celles redoutées avant son introduction. Il faut néanmoins souligner qu'une grande partie des responsables d'établissements de formation postgraduée et des médecins en formation ressentent dans leur pratique quotidienne les inconvénients du système DRG et, fait particulièrement inquiétant, constatent que le nouveau système ne tient pas compte de certains groupes de patients.

\section{Références}

1 Orlow P, Siegrist M, Giger M. Tendances observées dans la formation postgraduée médicale. Principaux résultats de l'enquête effectuée en 2006 auprès des médecins-assistants. Bulletin des médecins suisses 2007; 88(14/15):633-42. 